\title{
Generalized gradients on Lie algebroids
}

\author{
Bogdan Balcerzak · Antoni Pierzchalski
}

Received: 17 September 2012 / Accepted: 13 February 2013 / Published online: 20 March 2013

(C) The Author(s) 2013. This article is published with open access at Springerlink.com

\begin{abstract}
Generalized $O(n)$-gradients for connections on Lie algebroids are derived.
\end{abstract}
Keywords Lie algebroid · Connection · Stein-Weiss gradients · Young diagrams $\cdot$ Laplace type operators · Weitzenböck formulas

Mathematics Subject Classification (2010) Primary 58H05 - Secondary 17B10, 17B66, 53C05, 58A 10

\section{Introduction}

Gradients or generalized gradients in the sense of Stein and Weiss are first order differential operators that are irreducible summands of the covariant derivative $\nabla$. More exactly, if one starts from any vector bundle $E$ over $M$, a differential manifold, and terminates together with $\nabla$ in the bundle $T^{*} M \otimes E$ and if, additionally, one has a Lie group $G$ acting both on $T^{*} M$ and $E$ (and such a group is always strictly associated to the geometric structure considered on $M$ ), then one can think on splitting both the origin bundle $E$ and the target bundle $T^{*} M \otimes E$ onto direct sums of $G$-irreducible invariant subbundles. Then, the restriction of $\nabla$ to any one of such subbundles of $E$ composed with the projection onto any one of $T^{*} M \otimes E$ is just a $G$-gradient. We are mainly interested in $O(n)$-gradients, i.e., in the case $G=O(n)$. The exact definition is given in Sect. 2. Gradients are then the simplest bricks the covariant derivative is build of. $O(n)$-gradients were introduced first in 1968 by Stein and Weiss in their famous paper Generalization of the Cauchy-Riemann equations and representations of the rotation group [23]. Their theory developed next into a pretty large branch of global analysis, geometry,

B. Balcerzak

Institute of Mathematics, Lodz University of Technology, Wólczańska 215, 90-924 Łódź, Poland e-mail: bogdan.balcerzak@p.lodz.pl

A. Pierzchalski $(\bowtie)$

Faculty of Mathematics and Computer Science, University of Lodz, Banacha 22, 90-238 Łódź, Poland

e-mail: antoni@math.uni.lodz.pl 
differential operators or representation theory. Many natural first order linear differential operators in Riemannian geometry are either gradients or their compositions. For example, the exterior and interior derivatives $d$ and $\delta$, respectively, the Cauchy-Riemann operator $\bar{\partial}$ are gradients while the classical Dirac operator on exterior forms, namely, $d+\delta$ is their sum. Gradients depend on the geometry of $M$ (the group $G$ ) and this is obvious, but, on the other hand, they can themselves, e.g., by their spectral properties, determine, to some extent the geometry (volume, area of the boundary, scalar curvature), cf. [20,16,5,17]. The nice algebraic properties of gradients made that their theory can be successfully developed in the category of Lie algebroids.

A Lie algebroid over a manifold $M$ is a vector bundle $A$ over $M$ with a homomorphism of vector bundles $\varrho_{A}: A \rightarrow T M$ called an anchor, and a real Lie algebra structure $(\Gamma(A),[[\cdot, \cdot]])$ such that $\llbracket a, f \cdot b \rrbracket=f \cdot \llbracket a, b \rrbracket+\varrho_{A}(a)(f) \cdot b$ for all $a, b \in \Gamma(A)$, $f \in C^{\infty}(M)$. A Lie algebroid with a surjective anchor is called transitive. Any smooth manifold $M$ defines a Lie algebroid, where $A=T M$ with the identity anchor and the natural Lie algebra of vector fields on $M$. Other examples of Lie algebroids are: Lie algebras, integrable distributions, in particular foliations, cotangent bundles of Poisson manifolds, Lie algebroids of principal bundles. For more complete treatment of Lie algebroids and its connections we refer to [8,11-13] and [2].

The bundle $A$ is equipped here with a Riemannian metric $g$ extended then naturally to the whole tensor algebra over $A$.

Two important cases: the skew-symmetric forms and the trace-free symmetric tensors taken as the origin bundle are investigated in detail. In both of them the covariant derivative splits exactly into three pieces. One of our aim is getting a possibly full analogy and harmony in description of this two quite antipodal cases. The splittings coincide in the case of one tensors. Further similarities are as follows. Exactly one term of the splitting is-in both the cases - an elliptic operator in the sense that its symbol is injective. The suitable compositions of two terms lead to second order strongly elliptic operators: the Hodge-Laplacian $\Delta^{a}$ in the first case and an analogous differential operator $\Delta^{s}$ in the other. Both of them are, like $\nabla^{*} \nabla$, of metric symbol. As an application we derive the Weitzenböck type formulas in both the cases. It is interesting in its own that the zero order terms in the formulas are compositions of three summands in each case. The first is equivalent to the Ricci tensor in the classical case so it reflects the curvature of $\nabla$. The two others reflect a deviation of $\nabla$ from being metric and torsion-free, respectively.

It would be interesting to derive exact curvature terms in some particular cases and study their geometric meaning. It would be also interesting to investigate analogue problems for other geometric structures, in particular spinor one, what should lead to operators of the Dirac and the Rarita-Schwinger type.

\section{Connections and generalized gradients on Lie algebroids}

Let $\left(A, \varrho_{A}, \llbracket[\cdot, \cdot \mathbb{]})\right.$ be a Lie algebroid over a manifold $M$ and let $n$ be the dimension of the fiber of $A$. By an $A$-connection in a vector bundle $E$ we mean a homomorphism $\nabla: A \rightarrow$ $\mathcal{A}(E)$ of vector bundles $A$ and $\mathcal{A}(E)$, which commutes with anchors, and where $\mathcal{A}(E)$ is the Lie algebroid of the vector bundle $E$ (see [12]). The module $\mathcal{C D O}(E)$ of sections of $\mathcal{A}(E)$ is the space of all covariant differential operators in $E$, i.e. $\mathbb{R}$-linear operators $\ell: \Gamma(E) \rightarrow \Gamma(E)$ such that there is (unique) $X_{\ell} \in \mathfrak{X}(M)$ satisfying $\ell(f e)=f \ell(e)+$ $X_{\ell}(f) e$ for all $f \in C^{\infty}(M)$ and $e \in \Gamma(E)$. $\nabla$ determines a $\mathcal{C}^{\infty}(M)$-linear operator of modules of sections which will also be denoted by $\nabla$ and also called an A-connection. 
By the curvature of an $A$-connection $\nabla: \Gamma(A) \rightarrow \mathcal{C D O}(E)$ in $E$ we mean the 2-form $\mathcal{R}^{\nabla} \in \Gamma\left(\bigwedge^{2} A^{*} \otimes\right.$ End $\left.(E)\right)$ defined by

$$
\mathcal{R}_{a, b}^{\nabla}=\nabla_{a} \circ \nabla_{b}-\nabla_{b} \circ \nabla_{a}-\nabla_{\llbracket a, b \rrbracket}, \quad a, b \in \Gamma(A) .
$$

Notice that $\mathcal{R}^{\nabla}$ is zero if and only if $\nabla$ is a homomorphism of Lie algebras ( $\nabla$ preserves Lie brackets).

In the case where $E=A$, by a torsion of $\nabla$ we mean the 2-form $T^{\nabla} \in \Gamma\left(\bigwedge^{2} A^{*} \otimes A\right)$ given by

$$
T^{\nabla}(a, b)=\nabla_{a}(b)-\nabla_{b}(a)-\llbracket a, b \rrbracket, \quad a, b \in \Gamma(A) .
$$

If $T^{\nabla}=0, \nabla$ is called torsion-free.

Denote the vector bundles $\bigotimes^{k} A^{*}$ and $\otimes A^{*}$ by $A^{* \otimes k}$ and by $A^{* \otimes}$, respectively. Let $\bigwedge^{k} A^{*}$ and $S^{k} A^{*}$ denote the subbundles of skew-symmetric and symmetric $k$-tensors of $\otimes^{k} A^{*}$. Let

$$
\nabla: \Gamma(A) \longrightarrow \mathcal{C D O}(A)
$$

be an $A$-connection in the vector bundle $A$. Extend $\nabla$ in a natural way to the dual bundle by the formula

$$
\left(\nabla_{a} b^{*}\right)(c)=\varrho_{A}(a)\left(b^{*}(c)\right)-b^{*}\left(\nabla_{a} c\right)
$$

for all $a, c \in \Gamma(A), b^{*} \in \Gamma\left(A^{*}\right)$ and next by the Leibniz rule, to the whole tensor algebra of $A$. The extended connection will be denoted by the same symbol $\nabla$. In particular,

$$
\nabla_{a}(\zeta)\left(a_{1}, \ldots, a_{k}\right)=\varrho_{A}(a)\left(\zeta\left(a_{1}, \ldots, a_{k}\right)\right)-\sum_{j=1}^{k} \zeta\left(a_{1}, \ldots, \nabla_{a} a_{j}, \ldots, a_{k}\right)
$$

for $\zeta \in \Gamma\left(A^{* \otimes k}\right), a, a_{j} \in \Gamma(A)$. So, $\nabla$ can be treated as the differential operator $\nabla$ : $\Gamma\left(A^{* \otimes k}\right) \rightarrow \Gamma\left(A^{* \otimes k+1}\right)$ given by

$$
(\nabla \zeta)\left(a_{1}, a_{2} \ldots, a_{k+1}\right)=\left(\nabla_{a_{1}} \zeta\right)\left(a_{2}, \ldots, a_{k+1}\right)
$$

for $\zeta \in \Gamma\left(A^{* \otimes k}\right), a_{j} \in \Gamma(A)$.

\section{Lemma 2.1}

$$
\nabla \zeta=\sum_{j=1}^{n} a_{j}^{*} \otimes \nabla_{a_{j}} \zeta
$$

for any $\zeta \in \Gamma\left(A^{* \otimes}\right)$ and any dual local frames $\left(a_{1}, \ldots, a_{n}\right)$ of $A$ and $\left(a_{1}^{*}, \ldots, a_{n}^{*}\right)$ of $A^{*}$.

Proof Observe that

$$
\sum_{j=1}^{n} a_{j}^{*} \otimes \nabla_{a_{j}}: \Gamma\left(A^{* \otimes}\right) \longrightarrow \Gamma\left(A^{* \otimes}\right)
$$

is a derivation in the tensor algebra. So, it remains to show (2.5) for functions and sections of $A^{*}$. And this is just a calculation.

Define the second covariant derivative by

$$
\nabla_{a, b}^{2} \zeta=\nabla_{a}\left(\nabla_{b} \zeta\right)-\nabla_{\nabla_{a} b} \zeta, \quad \zeta \in \Gamma\left(A^{* \otimes}\right), a, b \in \Gamma(A) .
$$




\section{Lemma 2.2}

$$
\mathcal{R}_{a, b}^{\nabla} \zeta=\nabla_{a, b}^{2} \zeta-\nabla_{b, a}^{2} \zeta+\nabla_{T^{\nabla}(a, b)} \zeta
$$

for $\zeta \in \Gamma\left(A^{* \otimes}\right), a, b \in \Gamma(A)$.

Proof Simple consequence of (2.1), (2.2) and (2.6).

Suppose that $g$ is a Riemannian metric in $A$. $g$ defines in a usual manner the unique metric in $A^{*}$, which can be extended to the metric $\langle\cdot, \cdot\rangle_{g}$ in the vector bundle $A^{* \otimes k}$ by putting

$$
\left\langle\bigotimes_{i=1}^{k} a_{i}^{*}, \bigotimes_{j=1}^{k} b_{j}^{*}\right\rangle_{g}=\prod_{l=1}^{k}\left\langle a_{l}^{*}, b_{l}^{*}\right\rangle_{g}
$$

and next, by the restriction, to the metric in the bundles $\bigwedge^{k} A^{*}$ and $S^{k} A^{*}$, respectively. Notice that the metric in $\bigwedge^{k} A^{*}$ differs then by a multiplicative constant from that defined by the determinant: $\left\langle a_{1}^{*} \wedge \ldots \wedge a_{k}^{*}, b_{1}^{*} \wedge \ldots b_{k}^{*}\right\rangle_{g}=k ! \operatorname{det}\left(\left\langle a_{i}^{*}, b_{j}^{*}\right\rangle_{g}\right)$.

Now we are ready to define $O(n)$-gradients on $A$, where $n$ is the dimension of fibers. Since the fibres of $A$ are Euclidean spaces, $O(n)$ acts on them in a natural way. Obviously, the action can be extended to $A^{* \otimes}$. Decompose the space $A^{* \otimes k}$ into a direct sum of irreducible invariant subspaces:

$$
A^{* \otimes k}=\bigoplus_{\alpha} V_{\alpha}
$$

For every $\alpha$, denote by $j_{\alpha}: V_{\alpha} \rightarrow A^{* \otimes k}$ the natural injection defined by (2.8). Next, take any $\alpha$ and split the bundle $A^{*} \otimes V_{\alpha}$ into a direct sum

$$
A^{*} \otimes V_{\alpha}=\bigoplus_{\beta} W_{\beta}
$$

of invariant irreducible subbundles $W_{\beta}$. If the multiplicities are one-and it is the case of our considerations - this decomposition is unique. Denote by $\pi_{\beta}: A^{*} \otimes V_{\alpha} \rightarrow W_{\beta}$ the projections defined by the splitting (2.9).

For any $\alpha, \beta$ the first order differential operator

$$
\nabla^{\alpha \beta}=P_{\alpha \beta}=\pi_{\beta} \circ \nabla \circ j_{\alpha}: \Gamma\left(V_{\alpha}\right) \longrightarrow \Gamma\left(W_{\beta}\right)
$$

is called a generalized gradient or a Stein-Weiss operator.

\section{Theorem 2.1}

$$
\nabla^{\alpha \beta}=\pi_{\beta} \circ\left(\sum_{j=1}^{n} a_{j}^{*} \otimes \nabla_{a_{j}}\right) \circ j_{\alpha}
$$

for any dual local frames $\left(a_{1}, \ldots, a_{n}\right)$ of $A$ and $\left(a_{1}^{*}, \ldots, a_{n}^{*}\right)$ of $A^{*}$.

Proof Simple consequence of (2.10) and Lemma 2.1.

The simplest example is the case $k=1$. The origin bundle $A^{* \otimes 1}=A^{*}$ is irreducible $\left(O(n)\right.$ acts on $A^{*}$ transitively) but the target bundle $A^{* \otimes 2}$ splits into three $O(n)$-irreducible invariant subbundles:

$$
A^{* \otimes 2}=\bigwedge^{2} A^{*} \oplus \mathrm{S}_{o}^{k} A^{*} \oplus \mathrm{S}_{\mathrm{tr}}^{k} A^{*}
$$


where

$\bigwedge^{2} A^{*}$ is the subbundle of skew-symmetric tensors,

$\mathrm{S}_{o}^{k} A^{*}$ is the subbundle of symmetric and trace-free tensors,

$\mathrm{S}_{\mathrm{tr}}^{k} A^{*}$ is the subbundle of pure traces, i.e. tensors of the form $c g, c \in \mathbb{R}$.

Consequently, the three projections $\pi_{1}, \pi_{2}, \pi_{3}$ are given by:

$$
\pi_{1}=\frac{1}{2} \sum_{\sigma \in S_{2}} \operatorname{sgn} \sigma \sigma, \quad \pi_{2}=\frac{1}{2} \sum_{\sigma \in S_{2}} \sigma-\frac{1}{n} g \operatorname{tr}, \quad \pi_{3}=\frac{1}{n} g \text { tr },
$$

where the action of a permutation $\sigma \in S_{p}$ on a $p$-tensor $\zeta \in A^{* \otimes p}$ is given by

$$
(\sigma \zeta)\left(a_{1}, \ldots, a_{p}\right)=\zeta\left(a_{\sigma^{-1}(1)}, \ldots, a_{\sigma^{-1}(p)}\right),
$$

and $t r$ is the trace with respect to $g$. So, for the metric and torsion-free connection $\nabla$, the Stein-Weiss operators in this case are

$$
P_{1}=\pi_{1} \nabla=\frac{1}{2} d, \quad P_{2}=\pi_{2} \nabla=S, \quad P_{3}=\pi_{3} \nabla=-\frac{1}{n} g \delta
$$

and

$$
\nabla=\frac{1}{2} d+S-\frac{1}{n} g \delta
$$

where $d$ and $\delta$ are usual operators of exterior derivative and coderivative, respectively, defined generally later (cf. (3.1) and (3.4)), $S \alpha=\nabla^{S} \alpha+\frac{1}{n} \delta \alpha \cdot g, \alpha \in \bigwedge^{1} A^{*}\left(\nabla^{S}\right.$ is the symmetrized $\nabla)$, is known as the Cauchy-Ahlfors operator of $[1,18,19]$.

Come back to the general case. For any $k>1$ and any $\zeta \in \Gamma\left(A^{* \otimes k}\right)$ define its trace $\operatorname{tr} \zeta=\operatorname{tr}_{1,2} \zeta$, i.e. the trace with respect to the first two arguments by

$$
(\operatorname{tr} \zeta)\left(a_{1}, \ldots, a_{k-2}\right)=\sum_{j=1}^{n} \zeta\left(e_{j}, e_{j}, a_{1}, \ldots, a_{k-2}\right)
$$

where $\left(e_{1}, \ldots, e_{n}\right)$ is a local orthonormal frame of $A \cdot \operatorname{tr} \zeta=0$ for $\zeta \in \Gamma\left(A^{* \otimes 1}\right)$.

Define the coderivative operator

$$
\nabla^{*}: \Gamma\left(A^{* \otimes k}\right) \longrightarrow \Gamma\left(A^{* \otimes k-1}\right)
$$

by

$$
\nabla^{*} \zeta=-\operatorname{tr}(\nabla \zeta)
$$

In case $A$ is the tangent bundle of a Riemannian manifold $M$ the operator $\nabla^{*}$ reduces to the classical adjoint operator to the Levi-Civita $\nabla$. More exactly, $\nabla$ and $\nabla^{*}$ are there formally adjoint with respect to the global (integral) scalar product on $M$.

\section{Lemma 2.3}

$$
\nabla^{*} \zeta=-\sum_{j=1}^{n} i_{e_{j}}\left(\nabla_{e_{j}} \zeta\right)
$$

for any $\zeta \in \Gamma\left(A^{* \otimes}\right)$ and any local orthonormal frame $\left(e_{1}, \ldots, e_{n}\right)$ of $A$.

Proof One can easily see that the right-hand side of (2.15) is independent of choice of the frame. The rest of the proof is just a calculation. 
To understand the notion of gradients let us recall how tensors split under the action of the orthogonal group. The splitting can be explicitly described by the Young diagrams and by successive process of removing traces. We will follow here the construction of $O(n)$ irreducible splitting given by Weyl in [24] (cf. also [10] or [4]). For the sake of simplicity let us reduce our consideration to a single fibre of a bundle.

Let $E$ be a vector space over $\mathbb{R}$ of dimension $n$. Fix $p \in \mathbb{N}$ and take a sequence of integers $\alpha=\left(\alpha_{1}, \ldots, \alpha_{r}\right)$ with $\alpha_{1} \geq \cdots \geq \alpha_{r} \geq 1, \alpha_{1}+\cdots+\alpha_{r}=p$. Such an $\alpha$ is called a Young scheme of length $p$. It can be represented by a figure consisting of $r$ rows of squares such that the number of the squares in the $j$ th row is $\alpha_{j}$. A Young scheme can be filled with numbers $1, \ldots, p$ distributed in the squares in any order. A scheme filled with numbers is called a Young diagram.

Take a Young diagram $\alpha$ filled with numbers that grow both in rows and in columns. Denote by $H_{\alpha}$ and $V_{\alpha}$ the subgroup of the symmetric group $S_{p}$ consisting of all permutations preserving rows and columns, respectively. The diagram $\alpha$ determines the Young symmetrizer $\pi_{\alpha}: E^{\otimes p} \rightarrow E^{\otimes p}, E^{\otimes p}=\otimes^{p} E$, which is a linear operator given by

$$
\pi_{\alpha}=\sum_{\tau \in H_{\alpha}, \sigma \in V_{\alpha}} \operatorname{sgn} \sigma \cdot \tau \sigma
$$

Here the action of the permutation $\sigma \in S_{p}$ on tensors is defined in (2.11). It is known that $\pi_{\alpha}$ is a projection up to a multiplicative constant, i.e. $\pi_{\alpha}^{2}=m_{\alpha} \pi_{\alpha}$ for some $m_{\alpha} \in \mathbb{N}$ and that $E_{\alpha}=\operatorname{Im} \pi_{\alpha}$ is an invariant irreducible subspace of $E^{\otimes p}$ for the standard representation of $G L(n)$ in $E^{\otimes p}$. Moreover,

$$
E^{\otimes p}=\bigoplus_{\alpha} E_{\alpha}
$$

Assume that $E$ is equipped with a scalar product $g$. For $1 \leq i_{1}<i_{2} \leq p$ and $\zeta \in E^{\otimes p}$ define the $i_{1}, i_{2}$ th trace of $\zeta$ as the contraction of $g$ and $\zeta$ taken with respect to $i_{1}, i_{2}$ th arguments of $\zeta$. More exactly:

$$
\left(\operatorname{tr}_{i_{1}, i_{2}} \zeta\right)\left(v_{1}, \ldots, v_{p-2}\right)=\sum_{i=1}^{n} \zeta\left(v_{1}, \ldots, v_{i_{1}-1}, e_{i}, v_{i_{1}}, \ldots, v_{i_{2}-2}, e_{i}, v_{i_{2}-1}, \ldots, v_{p-2}\right)
$$

where $\left(e_{1}, \ldots, e_{n}\right)$ is an orthonormal frame of $E . \zeta$ is said to be trace-free if $\operatorname{tr}_{i_{1} i_{2}}(\zeta)=0$ for $1 \leq i_{1}<i_{2} \leq p$. It is obvious that the space $E_{0}^{\otimes p}$ of all trace-free tensors is a linear subspace of $E^{\otimes p} \cdot\left(E_{0}^{\otimes p}\right)^{\perp}$ is the subspace of all tensors of the form

$$
\sum_{\sigma \in S_{p}} \sigma\left(g \otimes \zeta_{\alpha}\right)
$$

where $\zeta_{\alpha} \in E^{\otimes p-2}$. The intersection $E_{\alpha 0}=E_{\alpha} \cap E_{0}^{\otimes p}$ is non-trivial if and only if the sum of the lengths of the first two columns of the Young diagram $\alpha$ is $\leq n$. Every such diagram is called admissible. The corresponding space $E_{\alpha 0}$ is invariant and irreducible under the $O(n)$-action and

$$
E_{0}^{\otimes p}=\bigoplus_{\alpha} E_{\alpha 0}
$$

where $\alpha$ ranges over the set of all admissible Young diagrams with numbers growing both in rows and columns. Using (2.16) and (2.17), and proceeding with the analogous 
decompositions of $E^{\otimes p-2}, E^{\otimes p-4}$, etc., one gets the decomposition of $E^{\otimes p}$ into the direct (in fact, orthogonal) sum of irreducible $O(n)$-invariant subspaces.

Among Young diagrams particularly interesting are that composed of a single column or of a single row only. In the first case $\alpha=(1,1, \ldots, 1), 1+1+\cdots+1=p, \frac{1}{p !} \pi_{\alpha}=$ Alt is the alternator. In the other one $\alpha=(p), \frac{1}{p !} \pi_{\alpha}=$ Sym is the symmetrizer. As a result the generalized gradients are then the terms of the covariant derivative acting on the space of skew-symmetric forms - in the first case, while they are the terms acting on the space of symmetric forms - in the other. These two important cases will be the subject of the next two sections.

All the considerations of the chapter apply of course to spaces of local sections of bundles considered below.

\section{Gradients on the skew-symmetric forms}

Let $\left(A, \varrho_{A}, \llbracket[\cdot, \cdot \rrbracket)\right.$ be a Lie algebroid over a manifold $M$ and let $\mathscr{A}=\bigoplus_{p \geq 0} \mathscr{A}^{k}$, where $\mathscr{A}^{k}=\Gamma\left(\bigwedge^{k} A^{*}\right)$, be the $C^{\infty}(M)$-module of skew-symmetric differential forms on the Lie algebroid $A . \mathscr{A}$ is an algebra over the ring $C^{\infty}(M)$ with the multiplication defined in the classical way:

$$
\wedge: \mathscr{A}^{p} \times \mathscr{A}^{q} \longrightarrow \mathscr{A}^{p+q}
$$

$(\omega \wedge \eta)\left(a_{1}, \ldots, a_{p+q}\right)=\sum_{\sigma \in S(p, q)} \operatorname{sgn} \sigma \cdot \omega\left(a_{\sigma(1)}, \ldots, a_{\sigma(p)}\right) \cdot \eta\left(a_{\sigma(p+1)}, \ldots, a_{\sigma(p+q)}\right)$,

where $S(p, q)$ is the set of $(p, q)$-shuffles. Let $\mu_{\omega}^{\wedge}: \mathscr{A}^{q} \rightarrow \mathscr{A}^{p+q}$ denote the operator of multiplication by $\omega \in \mathscr{A}^{p}$ given by

$$
\mu_{\omega}^{\wedge}(\eta)=\omega \wedge \eta \text { for } \eta \in \mathscr{A}^{q}
$$

Recall that the exterior derivative $d: \mathscr{A}^{k} \rightarrow \mathscr{A}^{k+1}$ (cf. [14]) is defined by

$$
\begin{aligned}
(d \eta)\left(a_{1}, \ldots, a_{k+1}\right)= & \sum_{j=1}^{k+1}(-1)^{j-1} \varrho_{A}\left(a_{j}\right)\left(\eta\left(a_{1}, \ldots \widehat{a}_{j} \ldots, a_{k+1}\right)\right) \\
& +\sum_{i<j}(-1)^{i+j} \eta\left(\llbracket a_{i}, a_{j} \rrbracket, a_{1}, \ldots \widehat{a}_{i} \ldots \widehat{a}_{j} \ldots, a_{k+1}\right) .
\end{aligned}
$$

Let $\nabla$ be an $A$-connection in $A$. Define the operator $d^{a}: \mathscr{A}^{k} \rightarrow \mathscr{A}^{k+1}$ by

$$
\left(d^{a} \eta\right)\left(a_{1}, \ldots, a_{k+1}\right)=\sum_{j=1}^{k+1}(-1)^{j-1}\left(\nabla_{a_{j}} \eta\right)\left(a_{1}, \ldots \widehat{a}_{j} \ldots, a_{k+1}\right) .
$$

A simple relation between $d$ and $d^{a}$ describes the following

\section{Lemma 3.1}

$$
d^{a}=d+d^{T}
$$

where

$$
\left(d^{T} \eta\right)\left(a_{1}, \ldots, a_{k+1}\right)=\sum_{i<j}(-1)^{i+j} \eta\left(T^{\nabla}\left(a_{i}, a_{j}\right), a_{1}, \ldots \widehat{a}_{i} \ldots \widehat{a}_{j} \ldots, a_{k+1}\right)
$$

for any $\eta \in \mathscr{A}^{k}, a_{1}, \ldots, a_{k+1} \in \Gamma(A)$. 
Notice that if $\nabla$ is torsion-free, $d^{a}=d$ (cf. also [3]). Notice also that in a general case $d^{a}$ is just - up to multiplicative constant-the alternation of $\nabla$. Namely,

$$
d^{a} \eta=(k+1) \cdot \operatorname{Alt}(\nabla \eta)
$$

where $\eta \in \mathscr{A}^{k}$, and where for any $\zeta \in A^{* \otimes p}$ its alternation Alt $\zeta$ is defined by

$$
\operatorname{Alt} \zeta=\frac{1}{p !} \sum_{\sigma \in S_{p}} \operatorname{sgn} \sigma(\sigma \zeta)
$$

Observe that $\nabla$ defined by (2.4) and restricted to $\mathscr{A}^{k}$ can be treated as the following operator $\nabla: \mathscr{A}^{k} \rightarrow \Gamma\left(A^{*} \otimes \bigwedge^{k} A^{*}\right)$.

By the exterior coderivative $d^{a *}$ we mean the restriction of the coderivative operator given in (2.14) to the space of skew-symmetric tensors:

$$
d^{a *}=\left.\nabla^{*}\right|_{\mathscr{A}^{k}}: \mathscr{A}^{k} \longrightarrow \mathscr{A}^{k-1} .
$$

Define two operators. The antisymmetric-trace

$$
\operatorname{tr}^{a}: \Gamma\left(A^{*} \otimes \bigwedge^{k} A^{*}\right) \longrightarrow \mathscr{A}^{k-1}
$$

as the restriction of the trace operator given in (2.13) to sections of $A^{*} \otimes \bigwedge^{k} A^{*}$ and the antisymmetric-cotrace

$$
\operatorname{cotr}^{a}: \mathscr{A}^{k-1} \longrightarrow \Gamma\left(A^{*} \otimes \bigwedge^{k} A^{*}\right)
$$

given by

$$
i_{b}\left(\operatorname{cotr}^{a} \eta\right)=\left(i_{b} g\right) \wedge \eta
$$

i.e. explicitly

$$
\left(\operatorname{cotr}^{a} \eta\right)\left(a_{0}, \ldots a_{k}\right)=\sum_{s=1}^{k}(-1)^{s-1} g\left(a_{0}, a_{s}\right) \cdot \eta\left(a_{1}, \ldots \widehat{a}_{s} \ldots a_{k}\right)
$$

for $\eta \in \mathscr{A}^{k-1}, b, a_{0}, \ldots a_{k} \in \Gamma(A)$.

Lemma 3.2 The operators $\operatorname{cotr}^{a} \eta$ and $k \cdot \operatorname{tr}^{a}$ are conjugate in the following sense $\operatorname{cotr}^{a}=$ $k \cdot\left(\operatorname{tr}^{a}\right)^{*}$ or more exactly:

$$
\left\langle\operatorname{cotr}^{a}(\eta), \xi\right\rangle_{g}=\left\langle\eta, k \cdot \operatorname{tr}^{a} \xi\right\rangle_{g}
$$

for $\eta \in \mathscr{A}^{k-1}, \xi \in \Gamma\left(A^{*} \otimes \bigwedge^{k} A^{*}\right)$. 
Proof Let $\eta \in \mathscr{A}^{k-1}, \alpha \in \Gamma\left(A^{*}\right), \zeta \in \mathscr{A}^{k},\left(e_{1}, \ldots, e_{n}\right)$ be an orthonormal local frame of A. Then

$$
\begin{aligned}
\langle\alpha & \left.\otimes \zeta, \operatorname{cotr}^{a}(\eta)\right\rangle_{g} \\
= & \sum_{i_{0}, \ldots, i_{k}=1}^{n} \alpha\left(e_{i_{0}}\right) \cdot \zeta\left(e_{i_{1}}, \ldots, e_{i_{k}}\right) \cdot \operatorname{cotr}^{a}(\eta)\left(e_{i_{0}}, \ldots, e_{i_{k}}\right) \\
= & \sum_{i_{0}, \ldots, i_{k}=1}^{n} \sum_{s=1}^{k} \alpha\left(e_{i_{0}}\right) \cdot \zeta\left(e_{i_{s}}, e_{i_{1}}, \ldots \widehat{e}_{i_{s}} \ldots, e_{i_{k}}\right) \cdot g\left(e_{i_{0}}, e_{i_{s}}\right) \cdot \eta\left(e_{i_{1}}, \ldots \widehat{e}_{i_{s}} \ldots, e_{i_{k}}\right) \\
= & \sum_{i_{1}, \ldots, i_{k}=1}^{n} \sum_{s=1}^{k} \alpha\left(e_{i_{s}}\right) \cdot \zeta\left(e_{i_{s}}, e_{i_{1}}, \ldots \widehat{e}_{i_{s}} \ldots, e_{i_{k}}\right) \cdot \eta\left(e_{i_{1}}, \ldots \widehat{e}_{i_{s}} \ldots, e_{i_{k}}\right) \\
= & \sum_{s=1}^{k} \sum_{i_{1}, \ldots \hat{i}_{s} \ldots, i_{k}=1}^{n} \operatorname{tr}^{a}(\alpha \otimes \zeta)\left(e_{i_{1}}, \ldots \widehat{e}_{i_{s}} \ldots, e_{i_{k}}\right) \cdot \eta\left(e_{i_{1}}, \ldots \widehat{e}_{i_{s}} \ldots, e_{i_{k}}\right) \\
& =k \sum_{i_{1}, \ldots, i_{k-1}=1}^{n} \operatorname{tr}^{a}(\alpha \otimes \zeta)\left(e_{i_{1}}, \ldots, e_{i_{k-1}}\right) \cdot \eta\left(e_{i_{1}}, \ldots, e_{i_{k-1}}\right) \\
& =\langle k \cdot \operatorname{tr}(\alpha \otimes \zeta), \eta\rangle_{g} .
\end{aligned}
$$

\section{Lemma 3.3}

$$
\operatorname{tr}^{a}\left(\operatorname{cotr}^{a} \eta\right)=(n-k+1) \eta
$$

for any $\eta \in \mathscr{A}^{k-1}$.

Proof Let $\eta \in \mathscr{A}^{k-1}, \alpha \in \Gamma\left(A^{*}\right), \zeta \in \mathscr{A}^{k},\left(e_{1}, \ldots, e_{n}\right)$ be an orthonormal local frame of $A, i_{2}, \ldots, i_{k} \in\{1, \ldots, n\}$. Then

$$
\begin{aligned}
& \operatorname{tr}\left(\operatorname{cotr}^{a} \eta\right)\left(e_{i_{2}}, \ldots, e_{i_{k}}\right)=\sum_{i_{1}=1}^{n}\left(\operatorname{cotr}^{a} \eta\right)\left(e_{i_{1}}, e_{i_{1}}, e_{i_{2}}, \ldots, e_{i_{k}}\right) \\
& =\sum_{i_{1}=1}^{n} \sum_{s=1}^{k}(-1)^{s-1} g\left(e_{i_{1}}, e_{i_{s}}\right) \eta\left(e_{i_{1}}, \ldots \widehat{e}_{i_{s}} \ldots, e_{i_{k}}\right) \\
& =\sum_{i_{1}=1}^{n} g\left(e_{i_{1}}, e_{i_{1}}\right) \eta\left(e_{i_{2}}, \ldots, e_{i_{k}}\right)+\sum_{i_{1}=1}^{n} \sum_{s=2}^{k}(-1)^{s-1} g\left(e_{i_{1}}, e_{i_{s}}\right) \eta\left(e_{i_{1}}, \ldots \widehat{e}_{i_{s}} \ldots, e_{i_{k}}\right) \\
& =n \cdot \eta\left(e_{i_{2}}, \ldots, e_{i_{k}}\right)+\sum_{s=2}^{k}(-1)^{s-1} g\left(e_{i_{s}}, e_{i_{s}}\right) \eta\left(e_{i_{s}}, e_{i_{2}}, \ldots \widehat{e}_{i_{s}} \ldots, e_{i_{k}}\right) \\
& =n \cdot \eta\left(e_{i_{1}}, \ldots, e_{i_{k}}\right)-(k-1) \cdot \eta\left(e_{i_{1}}, \ldots, e_{i_{k}}\right) \\
& =(n-k+1) \cdot \eta\left(e_{i_{1}}, \ldots, e_{i_{k}}\right) .
\end{aligned}
$$

Define three linear mappings

$$
\pi_{1}^{a}, \pi_{2}^{a}, \pi_{3}^{a}: \Gamma\left(A^{*} \otimes \bigwedge^{k} A^{*}\right) \longrightarrow \Gamma\left(A^{*} \otimes \bigwedge^{k} A^{*}\right)
$$


by

$$
\pi_{1}^{a}=\mathrm{Alt}, \quad \pi_{2}^{a}=\mathrm{id}-\pi_{1}^{a}-\pi_{3}^{a}, \quad \pi_{3}^{a}=\frac{1}{n-k+1} \operatorname{cotr}^{a} \circ \operatorname{tr}^{a} .
$$

\section{Lemma 3.4}

$$
\left\langle\pi_{1}^{a} \zeta, \pi_{3}^{a} \eta\right\rangle_{g}=\left\langle\pi_{1}^{a} \zeta, \pi_{2}^{a} \eta\right\rangle_{g}=\left\langle\pi_{2}^{a} \zeta, \pi_{3}^{a} \eta\right\rangle_{g}=0
$$

for $\zeta, \eta \in \Gamma\left(A^{*} \otimes \bigwedge^{k} A^{*}\right)$.

Proof Let $\zeta, \eta \in \Gamma\left(A^{*} \otimes \bigwedge^{k} A^{*}\right)$. Since $\pi_{1}^{a} \zeta$ and $\pi_{2}^{a} \zeta$ have zero traces, (3.5) implies at once that $\left\langle\pi_{1}^{a} \zeta, \pi_{3}^{a} \eta\right\rangle_{g}=0$ and $\left\langle\pi_{2}^{a} \zeta, \pi_{3}^{a} \eta\right\rangle_{g}=0$. Hence $\left\langle\pi_{1}^{a} \zeta, \pi_{2}^{a} \eta\right\rangle_{g}=\left\langle\pi_{1}^{a} \zeta, \eta\right\rangle_{g}-\left\langle\pi_{1}^{a} \zeta, \pi_{1}^{a} \eta\right\rangle_{g}$. If $\alpha \otimes \zeta_{o}, \beta \otimes \eta_{o} \in \Gamma\left(A^{*} \otimes \bigwedge^{k} A^{*}\right)$, then

$$
\begin{aligned}
& \left\langle\pi_{1}^{a}\left(\alpha \otimes \zeta_{o}\right), \beta \otimes \eta_{o}\right\rangle_{g}=\left\langle\text { Alt }\left(\alpha \otimes \zeta_{o}\right), \beta \otimes \eta_{o}\right\rangle_{g} \\
& \quad=\sum_{i_{0}, \ldots, i_{k}=1}^{n} \operatorname{Alt}\left(\alpha \otimes \zeta_{o}\right)\left(e_{i_{0}}, \ldots, e_{i_{k}}\right) \cdot\left(\beta \otimes \eta_{o}\right)\left(e_{i_{0}}, \ldots, e_{i_{k}}\right) \\
& \quad=\frac{1}{k+1} \sum_{i_{0}, \ldots, i_{k}=1}^{n} \sum_{s=0}^{k}(-1)^{s} \alpha\left(e_{i_{s}}\right) \cdot \zeta_{o}\left(e_{i_{0}}, \ldots \widehat{e}_{i_{s}} \ldots, e_{i_{k}}\right) \cdot \beta\left(e_{i_{0}}\right) \cdot \eta_{o}\left(e_{i_{1}}, \ldots, e_{i_{k}}\right) \\
& \quad=\left\langle\text { Alt }\left(\alpha \otimes \zeta_{o}\right), \text { Alt }\left(\beta \otimes \eta_{o}\right)\right\rangle_{g}=\left\langle\pi_{1}^{a}\left(\alpha \otimes \zeta_{o}\right), \pi_{1}^{a}\left(\beta \otimes \eta_{o}\right)\right\rangle_{g} .
\end{aligned}
$$

Consequently, $\left\langle\pi_{1}^{a} \zeta, \pi_{2}^{a} \eta\right\rangle_{g}=0$.

Theorem 3.1 $\pi_{1}^{a}, \pi_{2}^{a}, \pi_{3}^{a}$ are projections and $\Gamma\left(A^{*} \otimes \wedge^{k} A^{*}\right)$ splits onto the direct (in fact, orthogonal) sum of $O(n)$-invariant subspaces:

$$
\Gamma\left(A^{*} \otimes \bigwedge^{k} A^{*}\right)=\operatorname{Im} \pi_{1}^{a} \oplus \operatorname{Im} \pi_{2}^{a} \oplus \operatorname{Im} \pi_{3}^{a} .
$$

Proof The orthogonality of the splitting follows from Lemma 3.4. Clearly, $\pi_{1}^{a}$ is a projection. $\pi_{3}^{a}$ is a projection by Lemma 3.3. Since $\pi_{1}^{a}$ and $\pi_{3}^{a}$ are projections, $\pi_{2}^{a} \circ \pi_{2}^{a}=\pi_{2}^{a}+$ $\left(\pi_{1}^{a} \circ \pi_{3}^{a}+\pi_{3}^{a} \circ \pi_{1}^{a}\right)$. By the orthogonality, $\pi_{1}^{a} \circ \pi_{3}^{a}=0$ and $\pi_{3}^{a} \circ \pi_{1}^{a}=0$. So, $\pi_{2}^{a} \circ \pi_{2}^{a}=0$.

Remark 3.1 For $k \neq \frac{n}{4} \neq k+1$ the orthogonal subspaces in the splitting (3.7) are irreducible. For $n=4 k$ or $n=4(k+1)$ the origin bundle $\bigwedge^{\frac{n}{4}} A^{*}$ splits $\bigwedge_{+}^{\frac{n}{4}} A^{*} \oplus \bigwedge_{-}^{\frac{n}{4}} A^{*}$ where $+/-$ denotes the subbundles of $\bigwedge^{\frac{n}{4}} A^{*}$ being the eigenspaces of the Hodge star operator, respectively.

Notice that $\operatorname{Im} \pi_{1}^{a}=\mathscr{A}^{k+1}$. Elements of $\operatorname{Im} \pi_{1}^{a} \oplus \operatorname{Im} \pi_{2}^{a}$ are trace-free tensors, i.e. $\operatorname{tr}^{a} \eta=0$ for $\eta \in \operatorname{Im} \pi_{1}^{a} \oplus \operatorname{Im} \pi_{2}^{a}$, so elements of $\operatorname{Im} \pi_{3}^{a}$ may be called pure traces.

Define the Stein-Weiss type operators

$$
P_{j}^{a}=\pi_{j}^{a} \circ \nabla: \mathscr{A}^{k} \longrightarrow \Gamma\left(A^{*} \otimes \bigwedge^{k} A^{*}\right), \quad j \in\{1,2,3\} .
$$




\section{Theorem 3.2}

$$
\nabla=P_{1}^{a}+P_{2}^{a}+P_{3}^{a}
$$

Moreover,

$$
\begin{aligned}
& P_{1}^{a}=\frac{1}{k+1} d^{a}, \quad P_{2}^{a}=\nabla-\frac{1}{k+1} d^{a}+\frac{1}{n-k+1} \operatorname{cotr}^{a} \circ d^{a *}, \\
& P_{3}^{a}=\frac{-1}{n-k+1} \operatorname{cotr}^{a} \circ d^{a *} .
\end{aligned}
$$

Proof Simple consequence of (3.3), (3.4) and Theorem 3.1.

Remark 3.2 To get gradients in (3.7) for the exceptional cases, compose $P_{1}^{a}$ with the projections onto $\bigwedge_{+}^{\frac{n}{4}} A^{*}$ and $\bigwedge_{-}^{\frac{n}{4}} A^{*}$ for $n=4 k$, or restrict the origin bundle to one of $\bigwedge_{+}^{\frac{n}{4}} A^{*}$, $\bigwedge_{-}^{\frac{n}{4}} A^{*}$ for $n=4(k+1)$.

Notice that in the case $k=1$ the splitting of $\nabla$ reduces to that in (2.12) where $P_{1}^{a}=$ $P_{1}, P_{2}^{a}=P_{2}, P_{3}^{a}=P_{3}$.

Notice also that $P_{2}^{a}$ is the only elliptic operator of the three ones in the sense of injectivity of its symbol. For a linear operator $P: \Gamma(F) \rightarrow \Gamma(F)$ of order $m$ in a vector bundle $F$ its symbol at a given point $x \in M$ is defined by

$$
\sigma_{P}(\omega, e)=P\left(f^{m} \eta\right)(x)
$$

where $\omega \in A_{x}^{*}$ is such that $\omega=(d f)(x)$ for some smooth function $f$ satisfying $f(x)=$ $0, e \in F_{x}, \eta \in \Gamma(F)$ and $\eta(x)=e$ (cf. [15]); one can see that the definition is independent both of $f$ and of $\eta$ with $\eta(x)=e$. In our case $\sigma_{P_{1}^{a}}(\omega, \cdot)=\frac{1}{k+1} \mu_{\omega}^{\wedge}, \sigma_{d^{* a}}(\omega, \cdot)=-\operatorname{tr} \circ \mu_{\omega}^{\otimes}$. So, $\sigma_{P_{3}^{a}}(\omega, \cdot)=\frac{1}{n-k+1} \operatorname{cotr}^{a} \circ \operatorname{tr} \circ \mu_{\omega}^{\otimes}$, and

$$
\sigma_{P_{2}^{a}}(\omega, \cdot)=\mu_{\omega}^{\otimes}-\frac{1}{k+1} \mu_{\omega}^{\wedge}-\frac{1}{n-k+1} \operatorname{cotr}^{a} \circ \operatorname{tr} \circ \mu_{\omega}^{\otimes},
$$

where $\mu_{\omega}^{\otimes}$ is a tensor multiplication by $\omega$. One can check (cf. [18]) that if $A$ is transitive, the $\sigma_{P_{2}^{a}}(\omega, \cdot)$ is injective for all $\omega \neq 0$. The ellipticity of $P_{2}^{a}$ follows also from the general theory of gradients (cf. $[6,9,10])$.

\section{Weitzenböck formula for skew-symmetric tensors}

Define three differential operators of order zero. The Ricci type operator $\mathcal{R}^{a}: \mathscr{A} \rightarrow \mathscr{A}$ by

$$
\left(\mathcal{R}^{a} \eta\right)\left(a_{1}, \ldots, a_{k}\right)=\sum_{j=1}^{n} \sum_{s=1}^{k}(-1)^{s-1}\left(\mathcal{R}_{e_{j}, a_{s}}^{\nabla} \eta\right)\left(e_{j}, a_{1}, \ldots \widehat{a}_{s} \ldots, a_{k}\right)
$$

the operator $\mathcal{T}^{a}: \mathscr{A} \rightarrow \mathscr{A}$ by

$$
\left(\mathcal{T}^{a} \eta\right)\left(a_{1}, \ldots, a_{k}\right)=\sum_{j=1}^{n} \sum_{s=1}^{k}(-1)^{s-1}\left(\nabla_{T^{\nabla}\left(e_{j}, a_{s}\right)} \eta\right)\left(a_{1}, \ldots \widehat{a}_{s} \ldots, a_{k}\right)
$$


and next, the operator $\mathcal{M}^{a}: \mathscr{A} \rightarrow \mathscr{A}$ by

$$
\left(\mathcal{M}^{a} \eta\right)\left(a_{1}, \ldots, a_{k}\right)=\sum_{j=1}^{n} \sum_{s=1}^{k}(-1)^{s-1}\left(i_{\nabla_{a_{s}} e_{j}} i_{e_{j}}+i_{e_{j}} i_{\nabla_{a_{s}} e_{j}}\right)(\nabla \eta)\left(a_{1}, \ldots \widehat{a}_{s} \ldots, a_{k}\right)
$$

where $\eta \in \mathscr{A}^{k}, a_{1}, \ldots, a_{k} \in \Gamma(A),\left(e_{1}, \ldots, e_{n}\right)$ is a local orthonormal frame of $A, \mathcal{R}^{\nabla}$ is the curvature tensor of the connection $\nabla: \Gamma(A) \rightarrow \mathcal{C D O}\left(\bigwedge^{k} A^{*}\right)$. The first one $\mathcal{R}^{a}$ is like in the classical approach the trace of the curvature tensor. The next $\mathcal{T}^{a}$ reflects a deviation of the connection from being torsion-free. And the last $\mathcal{M}^{a}$ measures a non-compatibility of $\nabla$ with the metric. Notice that, by (2.7),

$$
\begin{aligned}
\left(\mathcal{R}^{a} \eta\right)\left(a_{1}, \ldots, a_{k}\right)= & \sum_{j=1}^{n} \sum_{s=1}^{k}(-1)^{s-1}\left(\nabla_{e_{j}, a_{s}}^{2} \eta-\nabla_{a_{s}, e_{j}}^{2} \eta\right)\left(e_{j}, a_{1}, \ldots \widehat{a}_{s} \ldots, a_{k}\right) \\
& +\left(\mathcal{T}^{a} \eta\right)\left(a_{1}, \ldots, a_{k}\right) .
\end{aligned}
$$

The Laplace operator on differential forms on the Lie algebroid $A$ is defined by

$$
\Delta^{a}=d^{a *} d^{a}+d^{a} d^{a *} .
$$

One can see that if $A$ is transitive, $\Delta^{a}$ is a second order strongly elliptic operator with the metric symbol $\sigma_{\Delta^{a}}(\omega, \eta)=|\omega|^{2} \eta$.

In much the same way as in [3] write the explicit formulas for the two summands of $\Delta$ :

$$
\begin{aligned}
\left(d^{*} d^{* a} \eta\right)\left(a_{1}, \ldots, a_{k}\right)= & -\operatorname{tr} \nabla^{2} \eta\left(a_{1}, \ldots, a_{k}\right) \\
& +\sum_{j=1}^{n} \sum_{s=1}^{k}(-1)^{s-1}\left(\nabla_{e_{j}, a_{s}}^{2} \eta\right)\left(e_{j}, a_{1}, \ldots \widehat{a}_{s} \ldots, a_{k}\right)
\end{aligned}
$$

and

$$
\begin{aligned}
\left(d^{a} d^{* *} \eta\right)\left(a_{1}, \ldots, a_{k}\right)= & -\left(\mathcal{M}^{a} \eta\right)\left(a_{1}, \ldots, a_{k}\right) \\
& -\sum_{j=1}^{n} \sum_{s=1}^{k}(-1)^{s-1}\left(\nabla_{a_{s}, e_{j}}^{2} \eta\right)\left(e_{j}, a_{1}, \ldots \widehat{a}_{s} \ldots, a_{k}\right)
\end{aligned}
$$

for any $\eta \in \mathscr{A}^{k}, a_{1}, \ldots, a_{k} \in \Gamma(A)$ and for any local orthonormal frame $\left(e_{1}, \ldots, e_{n}\right)$ of $A$. As a result we have the following

\section{Theorem 4.1 (Weitzenböck Formula)}

$$
\Delta^{a}=\nabla^{*} \nabla+\mathcal{R}^{a}-\mathcal{T}^{a}-\mathcal{M}^{a}
$$

where $\mathcal{R}^{a}, \mathcal{T}^{a}$ and $\mathcal{M}^{a}$ are the operators defined in (4.1)-(4.3).

Consider some particular cases. If $\nabla$ is metric, i.e. if

$$
\left(\varrho_{A} \circ a\right)(g(b, c))=g\left(\nabla_{a} b, c\right)+g\left(b, \nabla_{a} c\right) \text { for all } a, b, c \in \Gamma(A),
$$

the operator $\mathcal{M}^{a}$ vanishes.

If there exists a local orthonormal frame of sections $\left(e_{1}, \ldots, e_{n}\right)$ with the property $\left.\nabla_{e_{i}} e_{j}\right|_{x}=0$ at a single point $x \in M$ (for example if $A=F \subset T M$ is an integrable 
distribution on $M$ and $\nabla$ is the Levi-Civita connection), then $\mathcal{M}^{a}=0$. The assumption of existence of a local orthonormal frame of sections that have vanishing covariant derivatives at a single point implies that the isotropy algebra of $A$ (i.e. ker $\left.\varrho_{A}\right|_{X}$ ) is abelian, and consequently, $\mathcal{T}^{a}=0$.

If $\nabla$ is a torsion-free $A$-connection on $A$, then $d^{a}=d$ is the exterior derivative on $A$ given in (3.1), and obviously $\mathcal{T}^{a}=0$.

Notice additionally that if $\nabla$ is metric and $A$ is oriented, the Hodge star operator $*$ is well defined on $\mathscr{A}$, and then $d^{a *} \eta=(-1)^{n(p+1)+1} * d^{a} * \eta$ for any $\eta \in \mathscr{A}^{p}$ (cf. [3]).

\section{Gradients on the symmetric forms}

Let $\mathscr{S}^{k}$ be the $C^{\infty}(M)$-module of all symmetric differential forms, i.e. the module of sections of the symmetric subbundle $S^{k} A^{*}$ of $A^{* \otimes k}$ and $\mathscr{S}=\bigoplus_{k>0} \mathscr{S}^{k}$. Let $\mathscr{S}_{o}^{k}=$ $\left\{\omega \in \mathscr{S}^{k}: \operatorname{tr} \omega=0\right\}$ be the submodule of $\mathscr{S}^{k}$ of sections of the bundle $S_{o}^{k} A^{*}$ of all zero-trace tensors in $S^{k} A^{*}$, and let $\mathscr{S}_{o}=\bigoplus_{k \geq 0} \mathscr{S}_{o}^{k}$. Obviously, $\mathscr{S}$ is an algebra over the $\operatorname{ring} C^{\infty}(M)$ with the multiplication $\odot: \mathscr{S}^{p} \times \mathscr{S}^{q} \rightarrow \mathscr{S}^{p+q}$ given by

$$
(\zeta \odot \eta)\left(a_{1}, \ldots, a_{p+q}\right)=\sum_{\sigma \in S(p, q)} \zeta\left(a_{\sigma(1)}, \ldots, a_{\sigma(p)}\right) \cdot \eta\left(a_{\sigma(p+1)}, \ldots, a_{\sigma(p+q)}\right) .
$$

Let $\mu_{\zeta}^{\odot}: \mathscr{S}^{q} \rightarrow \mathscr{S}^{p+q}$ denote the operator of multiplication by $\zeta \in \mathscr{S}^{p}$ :

$$
\mu_{\zeta}^{\odot}(\eta)=\zeta \odot \eta \text { for } \eta \in \mathscr{S}^{q}
$$

The Lie algebroid structure on $A$ and the $A$-connection $\nabla$ determine the symmetric and $\mathbb{R}$-bilinear bracket $\{\cdot, \cdot\}: \Gamma(A) \times \Gamma(A) \rightarrow \Gamma(A)$ by

$$
\{a, b\}=-\left(\nabla_{a} b+\nabla_{b} a\right) .
$$

Observe that $\{a, f \cdot b\}=f \cdot\{a, b\}-\varrho_{A}(a)(f) \cdot b$ for all $a, b \in \Gamma(A)$ and $f \in C^{\infty}(M)$. In this way $A$ has the structure of a pseudo-Lie algebroid in the sense of [7] with the bracket $\{\cdot, \cdot\}$ and $-\varrho_{A}$ and $\varrho_{A}$ as the left and the right anchor, respectively.

Define the symmetric derivative $d: \mathscr{S}^{k} \rightarrow \mathscr{S}^{k+1}$ by

$$
\begin{aligned}
(\mathrm{d} \eta)\left(a_{1}, \ldots, a_{k+1}\right)= & \sum_{j=1}^{k+1} \rho_{A}\left(a_{j}\right)\left(\eta\left(a_{1}, \ldots \widehat{a}_{j} \ldots, a_{k+1}\right)\right) \\
& +\sum_{i<j} \eta\left(\left\{a_{i}, a_{j}\right\}, a_{1}, \ldots \widehat{a}_{i} \ldots \widehat{a}_{j} \ldots, a_{k+1}\right)
\end{aligned}
$$

and the operator $d^{s}: \mathscr{S}^{k} \rightarrow \mathscr{S}^{k+1}$ by

$$
\left(d^{s} \eta\right)\left(a_{1}, \ldots, a_{k+1}\right)=\sum_{j=1}^{k+1}\left(\nabla_{a_{j}} \eta\right)\left(a_{1}, \ldots \widehat{a}_{j} \ldots, a_{k+1}\right)
$$

for $\eta \in \mathscr{S}^{k}, a_{1}, \ldots, a_{k+1} \in \Gamma(A)$.

Observe that

$$
\mathrm{d}=d^{S}=(k+1) \cdot(\operatorname{Sym} \circ \nabla) \quad \text { on } \mathscr{S}^{k},
$$


where Sym is the symmetrizer given by

$$
(\operatorname{Sym} \vartheta)\left(a_{1}, \ldots, a_{k}\right)=\frac{1}{k !} \sum_{\sigma \in S_{k}} \vartheta\left(a_{\sigma(1)}, \ldots, a_{\sigma(k)}\right) \text { for all } \vartheta \in A^{* \otimes k} .
$$

By the symmetric coderivative $d^{S *}$ we mean the restriction of the coderivative operator given in (2.14) to the space of symmetric tensors:

$$
d^{s *}=\left.\nabla^{*}\right|_{\mathscr{S}^{k}}: \mathscr{S}^{k} \longrightarrow \mathscr{S}^{k-1} .
$$

Define two operators. The symmetric-trace

$$
\operatorname{tr}^{s}: \Gamma\left(A^{*} \otimes S^{k} A^{*}\right) \longrightarrow \mathscr{S}^{k-1}
$$

as the restriction of the trace operator given in (2.13) to $\Gamma\left(A^{*} \otimes S^{k} A^{*}\right)$ and the symmetriccotrace

$$
\operatorname{cotr}^{s}: \mathscr{S}^{k-1} \longrightarrow \Gamma\left(A^{*} \otimes \mathrm{S}^{k} A^{*}\right)
$$

given by

$$
i_{b}\left(\operatorname{cotr}^{s} \eta\right)=\left(i_{b} g\right) \odot \eta
$$

i.e. explicitly

$$
\left(\operatorname{cotr}^{s} \eta\right)\left(a_{0}, \ldots, a_{k}\right)=\sum_{s=1}^{k} g\left(a_{0}, a_{s}\right) \cdot \eta\left(a_{1}, \ldots \widehat{a}_{s} \ldots, a_{k}\right)
$$

for $\eta \in \mathscr{S}^{k-1}, b, a_{0}, \ldots a_{k} \in \Gamma(A)$.

\section{Lemma 5.1}

$$
\mu_{g}^{\odot}=\frac{k+1}{2} \operatorname{Sym} \circ \operatorname{cotr}^{s} \text { on } \mathscr{S}^{k-1}
$$

and

$$
i_{b} \circ \operatorname{cotr}^{s}=i_{b} \circ \mu_{g}^{\odot}-\mu_{g}^{\odot} \circ i_{b} \text { for every } b \in \Gamma(A) .
$$

Proof Let $\eta \in \mathscr{S}^{k-1}$ and $b, a_{0}, \ldots, a_{k} \in \Gamma(A)$. Since $\left(\mu_{g}^{\odot} \eta\right)\left(a_{0}, \ldots, a_{k}\right)=$ $\sum_{s<t} g\left(a_{s}, a_{t}\right) \cdot \eta\left(a_{0}, \ldots \widehat{a}_{s} \ldots \widehat{a}_{t} \ldots, a_{k}\right)$, we have

$$
\begin{aligned}
& (k+1) \operatorname{Sym}\left(\operatorname{cotr}^{s} \eta\right)\left(a_{0}, \ldots, a_{k}\right) \\
= & \sum_{s<t} g\left(a_{s}, a_{t}\right) \cdot \eta\left(a_{0}, \ldots \widehat{a}_{s} \ldots \widehat{a}_{t} \ldots, a_{k}\right) \\
& \quad+\sum_{t<s} g\left(a_{s}, a_{t}\right) \cdot \eta\left(a_{0}, \ldots \widehat{a}_{t} \ldots \widehat{a}_{s} \ldots, a_{k}\right) \\
= & 2 \sum_{s<t} g\left(a_{s}, a_{t}\right) \cdot \eta\left(a_{0}, \ldots \widehat{a}_{s} \ldots \widehat{a}_{t} \ldots, a_{k}\right) \\
= & 2\left(\mu_{g}^{\odot} \eta\right)\left(a_{0}, \ldots, a_{k}\right) .
\end{aligned}
$$

Moreover,

$$
i_{a}\left(\mu_{g}^{\odot} \eta\right)=i_{a}(g \odot \eta)=\left(i_{a} g\right) \odot \eta+g \odot\left(i_{a} \eta\right)=i_{a}\left(\operatorname{cotr}^{s} \eta\right)+\mu_{g}^{\odot}\left(i_{a} \eta\right) .
$$


Lemma 5.2 The operators $\operatorname{cotr}^{s} \eta$ and $k \cdot \operatorname{tr}^{s}$ are conjugate in the following sense $\operatorname{cotr}^{s}=$ $k \cdot\left(\operatorname{tr}^{s}\right)^{*}$ or more exactly:

$$
\left\langle\operatorname{cotr}^{s}(\eta), \zeta\right\rangle_{g}=\left\langle\omega, k \cdot \operatorname{tr}^{s} \zeta\right\rangle_{g}
$$

for $\eta \in \mathscr{S}^{k-1}, \zeta \in \Gamma\left(A^{*} \otimes \mathrm{S}^{k} A^{*}\right)$.

Proof Similar to the proof of (3.5) in Lemma 3.2.

\section{Lemma 5.3}

(a) $\operatorname{tr}^{s}\left(\operatorname{cotr}^{s} \eta\right)=(n+k-1) \eta$,

(b) $\operatorname{tr}^{s}\left(\mu_{g}^{\odot} \eta\right)=\mu_{g}^{\odot}\left(\operatorname{tr}^{s} \eta\right)+(n+2 k-2) \eta$

for any $\eta \in \mathscr{S}^{k-1}$.

Proof The proof of (a) is similar to that in the skew-symmetric case (Lemma 3.3). Let $\eta \in \mathscr{S}^{k-1},\left(e_{1}, \ldots, e_{n}\right)$ be an orthonormal local frame of $A, i_{2}, \ldots, i_{k} \in\{1, \ldots, n\}$. Then

$$
\begin{aligned}
\operatorname{tr}^{s} & \left(\mu{ }_{g}^{\odot} \eta\right)\left(e_{i_{2}}, \ldots, e_{i_{k}}\right) \\
= & \sum_{i_{1}=1}^{n} g\left(e_{i_{1}}, e_{i_{1}}\right) \cdot \eta\left(e_{i_{2}}, \ldots, e_{i_{k}}\right)+\sum_{i_{1}=1}^{n} \sum_{s=2}^{k} g\left(e_{i_{1}}, e_{i_{s}}\right) \cdot \eta\left(e_{i_{1}}, e_{i_{2}}, \ldots \widehat{e}_{i_{s}} \ldots, e_{i_{k}}\right) \\
& +\sum_{i_{1}=1}^{n} \sum_{t<s} g\left(e_{i_{t}}, e_{i_{s}}\right) \cdot \eta\left(e_{i_{1}}, e_{i_{1}}, e_{i_{2}}, \ldots \widehat{e}_{i_{t}} \ldots \widehat{e}_{i_{s}} \ldots, e_{i_{k}}\right) \\
= & n \cdot \eta\left(e_{i_{2}}, \ldots, e_{i_{k}}\right)+2 \sum_{s=2}^{k} g\left(e_{i_{s}}, e_{i_{s}}\right) \cdot \eta\left(e_{i_{s}}, e_{i_{2}}, \ldots \widehat{e}_{i_{s}} \ldots, e_{i_{k}}\right) \\
& +\sum_{t<s} g\left(e_{i_{t}}, e_{i_{s}}\right) \cdot\left(\operatorname{tr}^{s} \eta\right)\left(e_{i_{2}}, \ldots \widehat{e}_{i_{t}} \ldots \widehat{e}_{i_{s}} \ldots, e_{i_{k}}\right) \\
= & (n+2(k-1)) \cdot \eta\left(e_{i_{2}}, \ldots, e_{i_{k}}\right)+\mu \mu_{g}^{\odot}\left(\operatorname{tr}^{s} \eta\right)\left(e_{i_{2}}, \ldots, e_{i_{k}}\right) .
\end{aligned}
$$

Define the operator

$$
\pi_{\mathrm{tr}}=\frac{1}{n+k-1} \operatorname{cotr}^{s} \circ \operatorname{tr} \text { on } \Gamma\left(A^{*} \otimes \mathrm{S}_{o}^{k} A^{*}\right) .
$$

Lemma 5.3(a) implies that $\pi_{\mathrm{tr}}: \Gamma\left(A^{*} \otimes \mathrm{S}_{o}^{k} A^{*}\right) \rightarrow \Gamma\left(A^{*} \otimes \mathrm{S}_{o}^{k} A^{*}\right)$ is a projection. Moreover, since tr $\circ \pi_{\mathrm{tr}}=\operatorname{tr}$, then $\operatorname{tr} \circ\left(\mathrm{id}-\pi_{\mathrm{tr}}\right)=0$. Thus the operator id $-\pi_{\mathrm{tr}}$ is also a projection in $\Gamma\left(A^{*} \otimes \mathrm{S}_{o}^{k} A^{*}\right)$.

Now, define three linear mappings

$$
\pi_{1}^{s}, \pi_{2}^{s}, \pi_{3}^{s}: \Gamma\left(A^{*} \otimes \mathrm{S}_{o}^{k} A^{*}\right) \longrightarrow \Gamma\left(A^{*} \otimes \mathrm{S}_{o}^{k} A^{*}\right)
$$

by

$$
\pi_{1}^{s}=\operatorname{Sym} \circ\left(\mathrm{id}-\pi_{\mathrm{tr}}\right), \quad \pi_{2}^{s}=\mathrm{id}-\pi_{1}^{s}-\pi_{3}^{s}, \quad \pi_{3}^{s}=\pi_{\mathrm{tr}} .
$$

\section{Lemma 5.4}

$$
\left\langle\pi_{1}^{s} \zeta, \pi_{3}^{s} \eta\right\rangle_{g}=\left\langle\pi_{1}^{s} \zeta, \pi_{2}^{s} \eta\right\rangle_{g}=\left\langle\pi_{2}^{s} \zeta, \pi_{3}^{s} \eta\right\rangle_{g}=0
$$

for $\zeta, \eta \in \Gamma\left(A^{*} \otimes \mathrm{S}_{o}^{k} A^{*}\right)$. 
Proof Let $\zeta, \eta \in \Gamma\left(A^{*} \otimes \mathrm{S}_{o}^{k} A^{*}\right)$. Since $\pi_{1}^{s} \zeta$ and $\pi_{2}^{s} \zeta$ have zero traces, Lemma 5.2 implies at once that $\left\langle\pi_{1}^{s} \zeta, \pi_{3}^{s} \eta\right\rangle_{g}=0$ and $\left\langle\pi_{2}^{s} \zeta, \pi_{3}^{s} \eta\right\rangle_{g}=0$. Hence $\left\langle\pi_{1}^{s} \zeta, \pi_{2}^{s} \eta\right\rangle_{g}=\left\langle\pi_{1}^{s} \zeta, \eta\right\rangle_{g}-$ $\left\langle\pi_{1}^{s} \zeta, \pi_{1}^{s} \eta\right\rangle_{g}$. The proof of $\left\langle\pi_{1}^{s} \zeta, \eta\right\rangle_{g}=\left\langle\pi_{1}^{s} \zeta, \pi_{1}^{s} \eta\right\rangle_{g}$ is similar to a proper part of that of Lemma 3.4.

Theorem $5.1 \pi_{1}^{s}, \pi_{2}^{s}, \pi_{3}^{s}$ are projections and $\Gamma\left(A^{*} \otimes \mathrm{S}_{o}^{k} A^{*}\right)$ splits onto the direct (in fact, orthogonal) sum of $O(n)$-invariant subspaces:

$$
\Gamma\left(A^{*} \otimes \mathrm{S}_{o}^{k} A^{*}\right)=\operatorname{Im} \pi_{1}^{s} \oplus \operatorname{Im} \pi_{2}^{s} \oplus \operatorname{Im} \pi_{3}^{s} .
$$

Proof The orthogonality of the splitting follows from Lemma 5.4. Since Sym and $\pi_{\mathrm{tr}}$, id $-\pi_{\mathrm{tr}}$ are projections and Sym $\circ \pi_{\mathrm{tr}}=\pi_{\mathrm{tr}} \circ$ Sym, by Lemma 5.3(a) we obtain that $\pi_{1}^{s}$ and $\pi_{3}^{s}$ are projections in $\Gamma\left(A^{*} \otimes \mathrm{S}_{o}^{k} A^{*}\right)$. Consequently, $\pi_{2}^{s} \circ \pi_{2}^{s}=\pi_{2}^{s}+\left(\pi_{1}^{s} \circ \pi_{3}^{s}+\pi_{3}^{s} \circ \pi_{1}^{s}\right)$. But we see that

$$
\pi_{1}^{s} \circ \pi_{3}^{s}=\operatorname{Sym} \circ\left(\pi_{\mathrm{tr}}-\pi_{\mathrm{tr}} \circ \pi_{\mathrm{tr}}\right)=0 .
$$

Analogously $\pi_{3}^{s} \circ \pi_{1}^{s}=0$. Hence $\pi_{2}^{s}$ is a projection.

The space $\operatorname{Im} \pi_{3}^{s}$ is called the pure trace part of $\Gamma\left(A^{*} \otimes \mathrm{S}_{o}^{k} A^{*}\right)$ in the sense that $\operatorname{tr}_{i_{1}, i_{2}}\left(a^{*} \otimes \eta\right)=\pi_{3}^{s}$ for $i_{i}<i_{2}$. By Lemma $5.3 \pi_{1}^{s}, \pi_{2}^{s}, \pi_{3}^{s}$ can be explicitly expressed in the following forms:

$$
\begin{aligned}
& \pi_{1}^{s}=\operatorname{Sym}-\frac{2}{(n+k-1)(k+1)} \mu_{g}^{\odot} \circ \operatorname{tr}^{s}, \\
& \pi_{2}^{s}=\mathrm{id}-\operatorname{Sym}+\frac{2}{(n+k-1)(k+1)} \mu_{g}^{\odot} \circ \operatorname{tr}^{s}-\frac{1}{n+k-1} \operatorname{cotr}^{s} \circ \operatorname{tr}^{s}, \\
& \pi_{3}^{s}=\frac{1}{n+k-1} \operatorname{cotr}^{s} \circ \operatorname{tr}^{s} .
\end{aligned}
$$

Define the Stein-Weiss type operators

$$
P_{j}^{s}=\pi_{j}^{s} \circ \nabla: \mathscr{S}_{o}^{k} \longrightarrow \Gamma\left(A^{*} \otimes \mathrm{S}_{o}^{k} A^{*}\right), \quad j \in\{1,2,3\} .
$$

\section{Theorem 5.2}

$$
\nabla=P_{1}^{s}+P_{2}^{s}+P_{3}^{s}
$$

Moreover,

$$
\begin{aligned}
& P_{1}^{s} \zeta=\frac{1}{k+1}\left(d^{s} \zeta+\frac{2}{n+k-1} g \odot d^{s *} \zeta\right) \\
& P_{2}^{s} \zeta=\nabla \zeta-\frac{1}{k+1} d^{s} \zeta-\frac{2}{(n+k-1)(k+1)} g \odot d^{s *} \zeta+\frac{1}{n+k-1} \operatorname{cotr}^{s} d^{s *} \zeta \\
& P_{3}^{s} \zeta=\frac{-1}{n+k-1} \operatorname{cotr}^{s} \circ d^{s *} \zeta
\end{aligned}
$$

for $\zeta \in \mathscr{S}_{o}^{k}$.

Proof Simple consequence of (5.2), (5.3) and Theorem 5.1.

Remark 5.1 For $n \geq 5$ the orthogonal subspaces in (5.5) are irreducible, so, $P_{1}^{s}, P_{2}^{s}, P_{3}^{s}$ are gradients. If $n=4, P_{2}^{s}$ splits further on two $O(n)$-gradients. For $n=3$ the decomposition into irreducible parts is given by the Clebsch-Gordan formula. The details of the splitting in the two last cases can be found in [21]. 
Notice that in the case $k=1$ the splitting of $\nabla$ reduces to that in (2.12) where $P_{1}^{s}=$ $P_{2}, P_{2}^{s}=P_{1}, P_{3}^{s}=P_{3}$.

Notice also that $P_{1}^{s}$ is a symmetric counterpart of the Cauchy-Ahlfors operator investigated in $[1,21,22,18,19]$. Finally notice that similarly as in the skew-symmetric case only one $P_{1}^{S}$ is elliptic of the three considered gradients. Its symbol is equal to

$$
\sigma_{P_{1}^{s}}(\omega, \cdot)=\frac{1}{k+1} \mu_{\omega}^{\odot}+\frac{1}{n-k+1} \operatorname{Sym} \circ \operatorname{cotr}^{s} \circ \operatorname{tr} \circ \mu_{\omega}^{\otimes},
$$

since in our case $\sigma_{d^{s}}(\omega, \cdot)=\mu_{\omega}^{\odot}, \sigma_{d^{* a}}(\omega, \cdot)=-\operatorname{tr} \circ \mu_{\omega}^{\otimes}$, where $\mu_{\omega}^{\otimes}$ is a tensor multiplication by $\omega \in A_{x}^{*}$. Moreover,

$$
\sigma_{P_{3}^{s}}(\omega, \cdot)=\frac{1}{n+k-1} \operatorname{cotr}^{s} \circ \operatorname{tr} \circ \mu_{\omega}^{\otimes}, \quad \sigma_{P_{2}^{s}}(\omega, \cdot)=\mu_{\omega}^{\otimes}-\sigma_{P_{1}^{s}}(\omega, \cdot)-\sigma_{P_{3}^{s}}(\omega, \cdot) .
$$

One can check that if $A$ is transitive, $\sigma_{P_{1}^{s}}(\omega, \cdot)$ is injective for all $\omega \neq 0$. The ellipticity of $P_{1}^{s}$ follows also from the general theory of gradients (cf. $[10,9,6]$ ).

\section{Weitzenböck-type formula for symmetric forms}

Define the symmetric Ricci type operator $\mathcal{R}^{s}: \mathscr{S} \rightarrow \mathscr{S}$ by

$$
\left(\mathcal{R}^{s} \zeta\right)\left(a_{1}, \ldots, a_{k}\right)=\sum_{j=1}^{n} \sum_{s=1}^{k}\left(\mathcal{R}_{e_{j}, a_{s}}^{\nabla} \zeta\right)\left(e_{j}, a_{1}, \ldots \widehat{a}_{s} \ldots, a_{k}\right)
$$

the operator $\mathcal{T}^{s}: \mathscr{S} \rightarrow \mathscr{S}$ by

$$
\left(\mathcal{T}^{s} \zeta\right)\left(a_{1}, \ldots, a_{k}\right)=\sum_{j=1}^{n}\left(\nabla_{T^{\nabla}\left(e_{j}, a_{s}\right)} \zeta\right)\left(a_{1}, \ldots \widehat{a}_{s} \ldots, a_{k}\right)
$$

and next, $\mathcal{M}^{s}: \mathscr{S} \rightarrow \mathscr{S}$ by

$$
\left(\mathcal{M}^{s} \zeta\right)\left(a_{1}, \ldots, a_{k}\right)=\sum_{j=1}^{n} \sum_{s=1}^{k}\left(i_{\nabla_{a_{s}} e_{j}} i_{e_{j}}+i_{e_{j}} i_{\nabla_{a_{s}} e_{j}}\right)(\nabla \zeta)\left(a_{1}, \ldots \widehat{a}_{s} \ldots, a_{k}\right)
$$

where $\zeta \in \mathscr{S}^{k}, a_{1}, \ldots, a_{k} \in \Gamma(A),\left(e_{1}, \ldots, e_{n}\right)$ is a local orthonormal frame of $A, \mathcal{R}^{\nabla}$ is the curvature tensor of the connection $\nabla: \Gamma(A) \rightarrow \mathcal{C D O}\left(S^{k} A^{*}\right)$. Then, by Lemma 2.2,

$$
\begin{aligned}
\left(\mathcal{R}^{s} \zeta\right)\left(a_{1}, \ldots, a_{k}\right)= & \sum_{j=1}^{n} \sum_{s=1}^{k}\left(\nabla_{e_{j}, a_{s}}^{2} \zeta-\nabla_{a_{s}, e_{j}}^{2} \zeta\right)\left(e_{j}, a_{1}, \ldots \widehat{a}_{s} \ldots, a_{k}\right) \\
& +\left(\mathcal{T}^{s} \zeta\right)\left(a_{1}, \ldots, a_{k}\right) .
\end{aligned}
$$

\section{Theorem 6.1}

$-\left(d^{s *} d^{s} \eta\right)\left(a_{1}, \ldots, a_{k}\right)=\operatorname{tr} \nabla^{2} \eta\left(a_{1}, \ldots, a_{k}\right)+\sum_{j=1}^{n} \sum_{s=1}^{k}\left(\nabla_{e_{j}, a_{s}}^{2} \eta\right)\left(e_{j}, a_{1}, \ldots \widehat{a}_{s} \ldots, a_{k}\right)$

for $\eta \in \mathscr{S}^{k}, a_{1}, \ldots, a_{k} \in \Gamma(A)$. 
Proof Let $\eta \in \mathscr{S}^{k}, a_{1}, \ldots, a_{k} \in \Gamma(A)$. One can see that

$$
\begin{aligned}
\operatorname{tr} \nabla^{2} \eta\left(a_{1}, \ldots, a_{k}\right)= & \sum_{j=1}^{n} \nabla_{e_{j}}\left(\left(\nabla_{e_{j}} \eta\right)\left(a_{1}, \ldots, a_{k}\right)\right) \\
& -\sum_{j=1}^{n} \sum_{s=1}^{k}\left(\nabla_{e_{j}} \eta\right)\left(a_{1}, \ldots, \nabla_{e_{j}} a_{s}, \ldots, a_{k}\right) \\
& -\sum_{j=1}^{n}\left(\nabla_{\nabla_{e_{j}} e_{j}} \eta\right)\left(a_{1}, \ldots, a_{k}\right)
\end{aligned}
$$

and

$$
\begin{aligned}
& \left(\nabla_{e_{j}, a_{s}}^{2} \eta\right)\left(e_{j}, a_{1}, \ldots \widehat{a}_{s} \ldots, a_{k}\right) \\
= & \nabla_{e_{j}}\left(\left(\nabla_{a_{s}} \eta\right)\left(e_{j}, a_{1}, \ldots \widehat{a}_{s} \ldots, a_{k}\right)\right)-\left(\nabla_{a_{s}} \eta\right)\left(\nabla_{e_{j}} e_{j}, a_{1}, \ldots \widehat{a}_{s} \ldots, a_{k}\right) \\
& -\sum_{t \neq s}\left(\nabla_{a_{t}} \eta\right)\left(e_{j}, a_{1}, \ldots, \nabla_{e_{j}} a_{s}, \ldots \widehat{a}_{t} \ldots, a_{k}\right)-\left(\nabla_{\nabla_{e_{j}} a_{s}} \eta\right)\left(e_{j}, a_{1}, \ldots \widehat{a}_{s} \ldots, a_{k}\right) .
\end{aligned}
$$

Now, using definitions of $d^{s}, d^{s *}$, and collecting like terms one obtains the desired formula.

\section{Theorem 6.2}

$$
\left(d^{s} d^{s *} \eta\right)\left(a_{1}, \ldots, a_{k}\right)=\left(\mathcal{M}^{s} \eta\right)\left(a_{1}, \ldots, a_{k}\right)-\sum_{s=1}^{k} \sum_{j=1}^{n}\left(\nabla_{a_{s}, e_{j}}^{2} \eta\right)\left(e_{j}, a_{1}, \ldots \widehat{a}_{s} \ldots, a_{k}\right)
$$

for $\eta \in \mathscr{S}^{k}, a_{1}, \ldots, a_{k} \in \Gamma(A)$.

Proof Let $\eta \in \mathscr{S}^{k}, a_{1}, \ldots, a_{k} \in \Gamma(A)$. Since

$$
\begin{aligned}
\left(\nabla_{a_{s}, e_{j}}^{2} \eta\right)\left(e_{j}, a_{1}, \ldots \widehat{a}_{s} \ldots, a_{k}\right)= & \nabla_{a_{s}}\left(\left(\nabla_{e_{j}} \eta\right)\left(e_{j}, a_{1}, \ldots \widehat{a}_{s} \ldots, a_{k}\right)\right) \\
& -\left(\nabla_{e_{j}} \eta\right)\left(\nabla_{a_{s}} e_{j}, a_{1}, \ldots \widehat{a}_{s} \ldots, a_{k}\right) \\
& -\sum_{t \neq s}\left(\nabla_{e_{j}} \eta\right)\left(e_{j}, a_{1}, \ldots \widehat{a}_{s} \ldots \nabla_{a_{s}} a_{t} \ldots, a_{k}\right) \\
& -\left(\nabla_{\nabla_{a_{s}} e_{j}} \eta\right)\left(e_{j}, a_{1}, \ldots \widehat{a}_{s} \ldots, a_{k}\right),
\end{aligned}
$$

by (5.1) and (5.3) the theorem follows.

Define the Laplace-type operator on symmetric tensors by

$$
\Delta^{s}=d^{s *} d^{s}-d^{s} d^{s *} .
$$

One can check that if $A$ is transitive, $\Delta^{s}$ is a second order linear strongly elliptic operator with the metric symbol, i.e. for any covector $\omega$ and any symmetric $k$-tensor $\eta$ the symbol is given by the formula $\sigma_{\Delta^{s}}(\omega, \eta)=|\omega|^{2} \eta$.

As a consequence of theorems 6.1, 6.2, definitions of $\mathcal{T}^{s}, \mathcal{M}^{s}$ and (6.1) we obtain the following formula on symmetric tensors.

Theorem 6.3 (Weitzenböck-type Formula) $\Delta^{s}=\nabla^{*} \nabla-\mathcal{R}^{s}+\mathcal{T}^{s}-\mathcal{M}^{s}$.

Notice that if $\nabla$ is a metric $A$-connection, $\mathcal{M}^{s}=0$, and then $\Delta^{s}-\nabla^{*} \nabla=-\mathcal{R}^{s}+\mathcal{T}^{s}$. 
Open Access This article is distributed under the terms of the Creative Commons Attribution License which permits any use, distribution, and reproduction in any medium, provided the original author(s) and the source are credited.

\section{References}

1. Ahlfors, L.V.: Invariant operators and integral representations in hyperbolic spaces. Math. Scand. 36, 27-43 (1975)

2. Balcerzak, B., Kubarski, J., Walas, W.: Primary characteristic homomorphism of pairs of Lie algebroids and Mackenzie algebroid. Banach Center Publ. 54, 135-173 (2001)

3. Balcerzak, B., Kalina, J., Pierzchalski, A.: Weitzenböck formula on Lie algebroids. Bull. Polish Acad. Sci. Math. 60, 165-176 (2012)

4. Bartoszek, A., Kalina, J., Pierzchalski, A.: Gradients for $S L(q)$-foliations. J. Geom. Phys. 61, 2410-2416 (2011)

5. Branson, T., Gilkey, P.B., Ørsted, B., Pierzchalski, A.: Heat equation asymptotics of a generalized Ahlfors Laplacian on a manifold with boundary. In: Operator Theory: Advances and Applications, vol. 57, Birkhäuser Verlag, Basel, pp. 1-13 (1992)

6. Branson, T.: Stein-Weiss operators and ellipticity. J. Funct. Anal. 151, 334-383 (1997)

7. Grabowski, J., Urbański, P.: Lie algebroid and Poisson-Nijenhuis structure. Rep. Math. Phys. 40, 195-208 (1997)

8. Higgins, P.J., Mackenzie, K.C.H.: Algebraic constructions in the category of Lie algebroids. J. Algebra 129, 194-230 (1990)

9. Kalina, J., Ørsted, B., Pierzchalski, A., Walczak, P., Zhang, F.: Elliptic gradients and highest weights. Bull. Acad. Polon. Sci. Ser. Math. 44(4), 511-519 (1996)

10. Kalina, J., Pierzchalski, A., Walczak, P.: Only one of generalized gradients can be elliptic. Ann. Polon. Math. 67(2), 111-120 (1997)

11. Kosmann-Schwarzbach, Y., Laurent-Gengoux, C., Weinstein, A.: Modular classes of Lie algebroid morphisms. Transform. Groups 13, 727-755 (2008)

12. Mackenzie, K.C.H.: General Theory of Lie Groupoids and Lie Algebroids. London Mathematical Society Lecture Note Series 213, Cambridge University Press, Cambridge (2005)

13. Marle, C.-M.: Calculus on Lie algebroids, Lie groupoids and Poisson manifolds. Dissert. Math. 457, 1-57 (2008)

14. Maxim-Raileanu, L.: Cohomology of Lie algebroids. An. Sti. Univ. "Al. I. Cuza" Iasi Sect. I a Mat. (N.S.) 22(2), 197-199 (1976)

15. Narasimhan, R.: Analysis on Real and Complex Manifolds. North-Holland, 2nd edn. Springer- Verlag, New York (1985)

16. Ørsted, B., Pierzchalski, A.: The Ahlfors Laplacian on a Riemannian manifold. In: Constantin Caratheodory: an international tribute, 2, World Scientific, Teaneck, pp. 1021-1049 (1991)

17. Ørsted, B., Pierzchalski, A.: The Ahlfors Laplacian on a Riemannian manifold with boundary. Michigan Math. J. 43(1), 99-122 (1996)

18. Pierzchalski, A.: On quasiconformal deformations of manifolds and hypersurfaces. In: Proceedings of the Second Finnish-Polish Summer School in Complex Analysis, Jyväskylä, Bericht Univ. Jyvä skylä Math. Inst. 28, 79-94 (1984)

19. Pierzchalski, A.: Some differential operators connected with quasiconformal deformations on manifolds. In: Partial differential equations. Banach Center Publ. 19, 205-212 (1987)

20. Pierzchalski, A.: Ricci curvature and quasiconformal deformations of a Riemannian manifold. Manuscr. Math. 66, 113-127 (1989)

21. Reimann, H.M.: A rotation invariant differential equation for vector fields. Ann. Sc. Norm. Super. Pisa Cl. Sci. 9(4), 159-174 (1982)

22. Reimann, H.M.: Invariant system of differential operators. In: Proceeding of a seminar held in Torino May-June 1982, Topics in modern harmonic analysis, Instituto di alta Matematica, Roma (1983)

23. Stein, E., Weiss, G.: Generalization of the Cauchy-Riemann equations and representations of the rotation group. Amer. J. Math. 90, 163-196 (1968)

24. Weyl, H.: The Classical Groups. Princeton University Press, Princeton (1945) 